\title{
Singly resonant second-harmonic-generation frequency combs
}

\author{
T. Hansson,,${ }^{1, *}$ F. Leo, ${ }^{2}$ M. Erkintalo,,${ }^{3}$ S. Coen,${ }^{3}$ I. Ricciardi, ${ }^{4}$ M. De Rosa,${ }^{4}$ and S. Wabnitz ${ }^{5,4}$ \\ ${ }^{1}$ INRS-EMT, 1650 Boulevard Lionel-Boulet, Varennes, Québec J3X 1S2, Canada \\ ${ }^{2}$ OPERA-photonics, Université Libre de Bruxelles, 50 Avenue F. D. Roosevelt, CP 194/5, B-1050 Bruxelles, Belgium \\ ${ }^{3}$ The Dodd-Walls Centre for Photonic and Quantum Technologies, Department of Physics, \\ The University of Auckland, Auckland 1142, New Zealand \\ ${ }^{4}$ CNR-INO, Istituto Nazionale di Ottica, Via Campi Flegrei 34, I-80078 Pozzuoli (NA), Italy \\ ${ }^{5}$ Dipartimento di Ingegneria dell'Informazione, Università di Brescia, Via Branze 38, I-25123 Brescia, Italy
}

(Received 23 September 2016; published 3 January 2017)

\begin{abstract}
We consider frequency comb generation in dispersive singly resonant second-harmonic-generation cavity systems. Using a single temporal mean-field equation for the fundamental field that features a noninstantaneous nonlinear response function, we model the temporal and spectral dynamics and analyze comb generation, continuous wave bistability, and modulational instability. It is found that, owing to the significant temporal walk-off between the fundamental and second-harmonic fields, modulational instability can occur even in the complete absence of group-velocity dispersion. We further consider the relation of our model to a previously proposed modal expansion approach, and present a derivation of a general system of coupled mode equations. We show that the two models provide very similar predictions and become exactly equivalent in the limit that absorption losses and group-velocity dispersion at the fundamental frequency are neglected. Finally, we perform numerical simulations that show examples of the variety of comb states that are possible in phase-matched quadratic resonators, and discuss the dynamics of the comb formation process.
\end{abstract}

DOI: 10.1103/PhysRevA.95.013805

\section{INTRODUCTION}

Optical frequency combs have attracted a significant amount of interest in recent years. The ability to generate ultrabroadband combs with a uniform frequency spacing is widely seen as a breakthrough technology for creating a novel class of phase-coherent light sources that offer a wealth of potential applications in areas such as metrology and spectroscopy $[1,2]$. While early comb sources were based on mode-locked lasers, subsequent research has demonstrated the possibility of comb generation also in continuously pumped microresonator devices made of nonlinear materials, exploiting the intensity-dependent Kerr effect of the cubic $\chi^{(3)}$ nonlinearity $[3,4]$. This has allowed a reduction in size and an increase in efficiency of frequency comb sources to such a degree that it may soon be possible to create fully integrated chip-scale comb synthesizers.

Very recently, an alternative approach for comb generation has emerged, based on direct comb formation in quadratic $\chi^{(2)}$ nonlinear media [5-7]. In particular, by pumping a cavity containing a quadratic nonlinear crystal that is phase matched for second-harmonic generation, frequency combs have simultaneously been observed around both fundamental and second-harmonic wavelengths [7]. Quadratic comb sources are interesting for several reasons. They could, e.g., reduce the power requirements for comb generation when compared with Kerr combs, due to the inherently higher strength of the quadratic nonlinearity. Moreover, they could allow the transfer of comb lines to new spectral regions, either where they are prevented by dispersion, such as in the visible, or where the availability of suitable pump sources is limited, such as in the mid-infrared spectral range.

\footnotetext{
*tobias.hansson@emt.inrs.ca
}

The generation mechanism of quadratic combs relies on cascaded $\chi^{(2)}$ processes that resemble an effective $\chi^{(3)}$ nonlinearity (not necessarily of the Kerr type), which allow for the generation of sidebands above a certain power threshold through a subharmonically pumped optical parametric oscillation (OPO) process [7-9]. In quadratic comb generation, pump photons are first converted through second-harmonic generation (SHG) from the fundamental field to the second harmonic, before being down-converted back to signal-idler sidebands through nondegenerate OPO. Remarkably, this process has been found to occur not only in the presence of a large phase mismatch, a condition that is well known to produce an effective Kerr (or cascading) nonlinearity in quadratic media [10], but also with perfect phase matching [7]. The reason is the presence of a large temporal walk-off, that arises due to the difference in group velocities between the fundamental and the second-harmonic fields. The walk-off occurs in dispersive SHG cavities, and underpins the dynamics by inhibiting competing sum-frequency generation (SFG) processes [11]. The result is the generation of sidebands around both fundamental and second-harmonic frequencies through a modulational instability process which bears resemblance to that occurring in cubic Kerr media. After their formation, the subharmonic fields can subsequently interact to create new frequency components through different cascaded second-order processes, resulting in the generation of two broad and equally spaced frequency combs around the fundamental and the second harmonic.

The magnitude of the walk-off, in combination with the presence of higher-order dispersion, is in fact a distinguishing feature of temporally dispersive cavities with respect to more widely studied spatially diffractive SHG cavities [12], and results in the observation of different dynamics for the two types of systems. Moreover, the dynamics in cavity SHG is also fundamentally different when compared to SHG generation in single pass systems [13], owing to the resonant nature of the 
cavity configuration and the presence of boundary conditions that result in a dissipative system driven by the external pump laser.

In the following, we will limit the discussion to singly resonant SHG [11], where only frequencies around the fundamental field are resonant, while the second-harmonic field leaves the cavity at the end of each roundtrip. Other configurations such as doubly resonant SHG have recently been studied elsewhere [14], and a corresponding more detailed analysis for those configurations will be the subject of future work. In the next section, we will introduce a theoretical formalism for describing the temporal dynamics of quadratic frequency combs. We show that, for singly resonant conditions, a single mean-field equation can be derived which is analogous to the Lugiato-Lefever equation for Kerr media [15,16], however with an effective noninstantaneous $\chi^{(3)}$ nonlinearity. The significance of this mean-field equation is that it provides a simple model that captures the essential physics of SHG frequency comb generation, speeds up numerical simulations, and permits analytical investigations.

The temporal mean-field model was first presented by the authors in Ref. [11]. In this paper, we consider an extension of the model that includes higher-order dispersion and perform an analysis of both the continuous wave (CW) bistability and the modulational instability (MI). Moreover, we demonstrate that the coupled mode equations previously proposed in Ref. [7] can be derived using a modal expansion approach, thus proving explicitly the connection between the temporal and frequency domain formalisms. In fact, we find that the two approaches are only approximately equivalent in the meanfield limit, due to subtle differences in the averaging procedure. Finally, we perform numerical simulations which show some of the rich spectral dynamics that can be observed across the whole parameter space, and reveal the dynamics underpinning the formation of temporal patterns corresponding to phaselocked quadratic optical frequency combs.

\section{THE MEAN-FIELD MODEL}

A resonant cavity containing a quadratic nonlinear medium can, under quite general circumstances, be modeled by using an infinite-dimensional map of evolution equations for the fundamental field (FF) and second-harmonic (SH) field, together with boundary conditions that relate the fields between different roundtrips. We take $A_{m}(z, \tau)$ and $B_{m}(z, \tau)$ to be the slowly varying envelopes of the electric field in units of $[\sqrt{W}]$ at the fundamental $\left(\omega_{0}\right)$ and second-harmonic $\left(2 \omega_{0}\right)$ frequency, respectively. Propagation through the $m$ th roundtrip of the quadratic medium of length $L$ is then described by the two coupled equations $[11,17]$,

$$
\begin{aligned}
& \frac{\partial A_{m}}{\partial z}=\left[-\frac{\alpha_{c 1}}{2}+i D_{1}\left(i \frac{\partial}{\partial \tau}\right)\right] A_{m}+i \kappa B_{m} A_{m}^{*} e^{-i \Delta k z} \\
& \frac{\partial B_{m}}{\partial z}=\left[-\frac{\alpha_{c 2}}{2}-\Delta k^{\prime} \frac{\partial}{\partial \tau}+i D_{2}\left(i \frac{\partial}{\partial \tau}\right)\right] B_{m}+i \kappa A_{m}^{2} e^{i \Delta k z},
\end{aligned}
$$

where $z$ is the longitudinal coordinate and $\tau$ is a (fast) time variable in a reference frame moving at the group velocity of the fundamental frequency. Dispersion is included

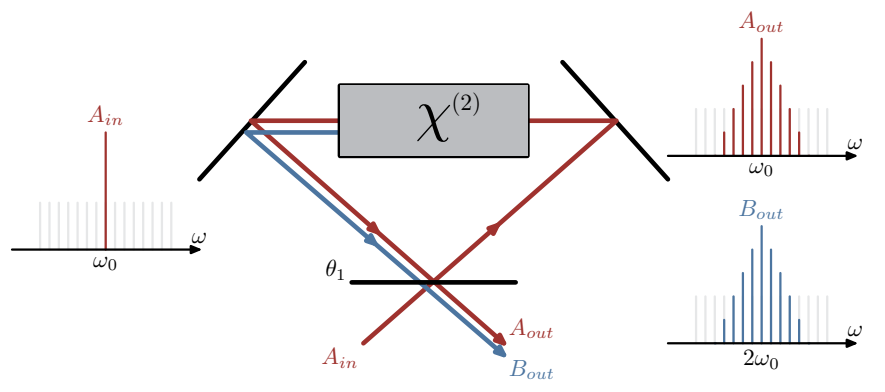

FIG. 1. Schematic example of a singly resonant SHG cavity system. A cavity containing a medium with a $\chi^{(2)}$ nonlinearity is driven by a $\mathrm{CW}$ field $A_{\text {in }}$ at frequency $\omega_{0}$. The resonant fundamental field $A$ generates a nonresonant second-harmonic field $B$ at frequency $2 \omega_{0}$ that leaves the cavity at each roundtrip. Modulational instability due to temporal walk-off leads to frequency combs being generated at cavity modes around both $\mathrm{FF}$ and $\mathrm{SH}$ frequencies.

in the model, to all orders, through the two operators $D_{1,2}(x)=\sum_{l \geqslant 2}\left(k_{1,2}^{(l)} / l !\right) x^{l}$, with $k_{1,2}^{(l)}=d^{l} k /\left.d \omega^{l}\right|_{\omega_{0}, 2 \omega_{0}}$ being dispersion coefficients obtained by Taylor series expansion of the propagation constants. In Eqs. (1) and (2) we have defined $\Delta k=2 k_{1}-k_{2}$ as the wave-vector mismatch for the SHG process, while $\Delta k^{\prime}=k_{2}^{(1)}-k_{1}^{(1)}$ is the group-velocity mismatch between the fundamental and second-harmonic fields. The nonlinear coefficient is given by $\kappa=\sqrt{8} \omega_{0} \chi_{\text {eff }}^{(2)} / \sqrt{c^{3} n_{1}^{2} n_{2} \epsilon_{0}}$, where $\chi_{\mathrm{eff}}^{(2)}$ is the effective second-order susceptibility, $c$ the speed of light, $n_{1,2}$ are refractive indices evaluated at the $\mathrm{FF}$ and $\mathrm{SH}$, respectively, and $\epsilon_{0}$ is the vacuum permittivity. Additionally, the absorption coefficients are $\alpha_{c 1,2}$, the power coupling coefficients are $\theta_{1,2}$ and the phase detunings are $\delta_{1,2}$ for each of the two fields, respectively. The map is completed by requiring that the fields should satisfy the following cavity boundary conditions,

$$
\begin{gathered}
A_{m+1}(0, \tau)=\sqrt{1-\theta_{1}} A_{m}(L, \tau) e^{-i \delta_{1}}+\sqrt{\theta_{1}} A_{\text {in }}, \\
B_{m+1}(0, \tau)=\sqrt{1-\theta_{2}} B_{m}(L, \tau) e^{-i \delta_{2}} .
\end{gathered}
$$

As we are here considering SHG, the resonator is assumed to be driven at the FF by the external continuous wave field $A_{\text {in. }}$. The map Eqs. (1)-(4) can be used to model degenerate intracavity SHG and OPO [with a change of driving term to Eq. (4)] for a wide range of experimentally relevant circumstances. However, a more general model is necessary for describing ultrabroadband fields with multiple harmonics or where the fundamental and second harmonic are partially overlapping [18].

We now focus on singly resonant SHG, where only the fundamental field is resonant. A schematic of an example system is illustrated in Fig. 1. The buildup of the SH field occurs only during a single pass of the nonlinear crystal, with the $\mathrm{SH}$ field leaving the cavity at each roundtrip without being reinjected, i.e., $\theta_{2}=1$. This corresponds to the initial condition $B_{m}(0, \tau)=0$, and implies that the $\mathrm{SH}$ field will be slaved to the fundamental. Under these conditions it becomes possible to reduce the map to a single temporal mean-field equation [11]. The derivation is as follows. Equation (2) is first solved 
formally using the Fourier transform to obtain the solution,

$$
\mathscr{F}\left[B_{m}(z, \tau)\right]=i \kappa e^{\hat{k} z} \int_{0}^{z} e^{(i \Delta k-\hat{k}) z^{\prime}} \mathscr{F}\left[A_{m}^{2}\left(z^{\prime}, \tau\right)\right] d z^{\prime},
$$

where $\mathscr{F}[\cdot]=\int_{-\infty}^{\infty} \cdot e^{i \Omega \tau} d \tau$ and $\hat{k}(\Omega)=-\alpha_{c 2} / 2+i\left[\Delta k^{\prime} \Omega+\right.$ $\hat{D}_{2}(\Omega)$ ] (the hat signifies a function defined in the Fourier domain). The inverse transform of this result is next used to eliminate the SH field $B_{m}$ from Eq. (1), which then yields a single evolution equation for the fundamental field, viz.

$$
\begin{aligned}
\frac{\partial A_{m}}{\partial z}= & {\left[-\frac{\alpha_{c 1}}{2}+i D_{1}\left(i \frac{\partial}{\partial \tau}\right)\right] A_{m}-\kappa^{2} A_{m}^{*} } \\
& \times \mathscr{F}^{-1}\left[\int_{0}^{z} e^{(\hat{k}-i \Delta k)\left(z-z^{\prime}\right)} \mathscr{F}\left[A_{m}^{2}\left(z^{\prime}, \tau\right)\right] d z^{\prime}\right] .
\end{aligned}
$$

Equation (6) can be used, together with the boundary condition Eq. (3), to model the evolution of the FF, but considerable simplification can be obtained by using the mean-field approximation. Here the FF is assumed to evolve slowly enough during each roundtrip, so that its $z$ variation can be neglected to the first order. By assuming the coupling coefficient $\theta_{1}$ and the detuning $\delta_{1}$ to be quantities of the first order, and averaging Eq. (6) over the roundtrip length [19], we obtain a mean-field equation in the slow time variable $t\left(A\left(t=m t_{R}, \tau\right)=A_{m}(z=0, \tau)\right)$, viz.

$$
\begin{aligned}
t_{R} \frac{\partial A(t, \tau)}{\partial t}= & {\left[-\alpha_{1}-i \delta_{1}+i L D_{1}\left(i \frac{\partial}{\partial \tau}\right)\right] A } \\
& -\rho A^{*}\left[A^{2}(t, \tau) \otimes I(\tau)\right]+\sqrt{\theta_{1}} A_{\text {in }},
\end{aligned}
$$

where $t_{R}$ is the roundtrip time, $\alpha_{1}=\left(\alpha_{c 1} L+\theta_{1}\right) / 2$ the roundtrip loss, $\rho=(\kappa L)^{2}, \otimes$ denotes convolution, and $I(\tau)=$ $\mathscr{F}^{-1}[\hat{I}(\Omega)]$ is a nonlinear response function given by

$$
\hat{I}(\Omega)=\frac{1}{L^{2}} \int_{0}^{L} \int_{0}^{z} e^{(\hat{k}-i \Delta k)\left(z-z^{\prime}\right)} d z^{\prime} d z=\frac{1-e^{-i x}-i x}{x^{2}},
$$

with $x(\Omega)=[\Delta k+i \hat{k}(\Omega)] L$. It is often useful to work with a normalized form of the equation; see Appendix.

The mean-field Eq. (7) is seen to exhibit an effective third-order nonlinearity with a noninstantaneous response that is resemblant of the delayed Raman response found in cubic Kerr media [20] and other noninstantaneous nonlinear Schrödinger models [21]. However, contrary to those models there is also a phase dependence, since the square of the field, rather than the intensity, is involved. In the case that losses at the $\mathrm{SH}$ can be neglected, the argument $x(\Omega)$ is purely real and the response function can be written as $\hat{I}(\Omega)=(1 / 2) \operatorname{sinc}^{2}(x / 2)+i(1 / x)(\operatorname{sinc}(x)-1)$. The response function, shown in Fig. 2, can be interpreted as representing a complex coupling coefficient for an SFG process between the pump and a signal wave for the interaction $\omega_{0}, \omega_{0}+\Omega \rightarrow$ $2 \omega_{0}+\Omega$. The nature of the interaction and the direction of energy flow depends on the relative phases between the FF and SH fields, and is determined by the response function whose real part represents a frequency-dependent nonlinear loss, akin
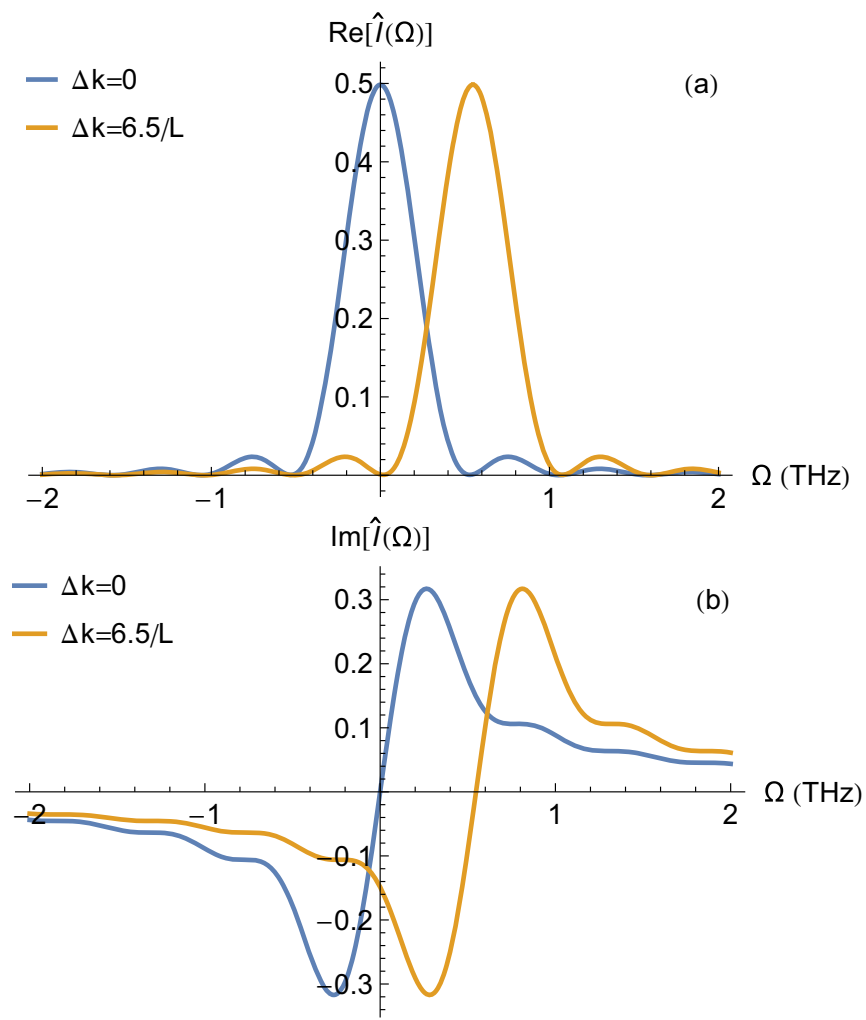

FIG. 2. Real (a) and imaginary (b) part of the (dimensionless) nonlinear response function $\hat{I}(\Omega)$ with and without phase mismatch. Parameters corresponding to a $\mathrm{LiNbO}_{3}$ crystal: $\Delta k^{\prime}=792 \mathrm{ps} / \mathrm{m}$, $k_{2}^{(2)}=0.714 \mathrm{ps}^{2} / \mathrm{m}, \alpha_{c 2}=0$, and $L=15 \mathrm{~mm}$.

to that due to two-photon absorption or to the presence of a saturable absorber, while the imaginary part represents a phase modulation.

In Fig. 2 we have plotted the response function both with and without a finite phase mismatch $\Delta k$. In the latter, phase matched, case a signal mode at $\Omega=0$ experiences a pure loss; while in the former, phase mismatched, case we find that the effect of the nonlinearity is primarily to provide self-phase modulation of the field. The relative contributions of the two effects depend on the size of the mismatch. Self-phase modulation is also seen to occur at the pump frequency with a sign that is conditional on that of the phase mismatch. Assuming that the linear absorption loss at the second harmonic is small, we find that nonlinear losses have minima for $x \approx 2 \pi n$ with $n$ being an integer different from zero. These minima correspond to frequencies $\Omega_{n} \approx(\Delta k L+2 \pi n) /\left(\Delta k^{\prime} L\right)$ and coincide with spectral positions at which sidebands are likely to develop. Conversely, the peak of the nonlinear loss occurs for $n=0$.

Using Cauchy's integral theorem, it can be demonstrated that the time domain response $I(\tau)$ disappears outside of $0 \leqslant$ $\tau \lesssim \Delta k^{\prime} L$ for $\Delta k^{\prime}>0$ (or $\Delta k^{\prime} L \lesssim \tau \leqslant 0$ for $\Delta k^{\prime}<0$ ), since the integrand of the inverse Fourier transform is an analytic function outside of this region. The walk-off thus determines whether or not a memory effect is present and the duration of the noninstantaneous response. Finally, we remark that the SH field is slaved to the fundamental in accordance with Eq. (5), from which we can obtain an expression for the output field at 


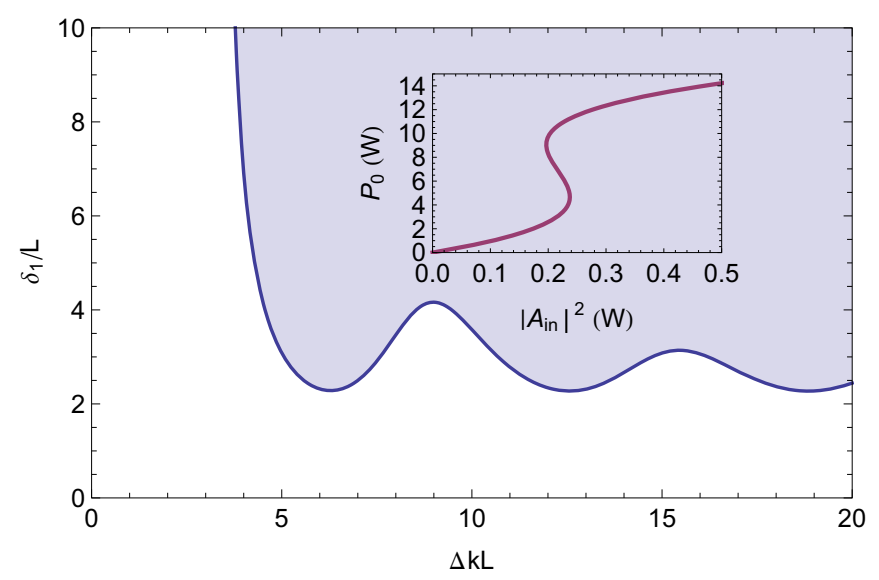

FIG. 3. Bistability regions as a function of phase mismatch and detuning. Unshaded and shaded regions correspond to parameter ranges where the stationary $\mathrm{CW}$ solution can have either one or three simultaneous real solutions, respectively. Inset shows bistability of intracavity power for $\delta_{1} / L=3$ and $\Delta k L=6.5$.

the second harmonic, viz.

$$
B(L, \tau)=i \kappa \mathscr{F}^{-1}\left[\frac{e^{\hat{k} L}-e^{i \Delta k L}}{\hat{k}-i \Delta k} \mathscr{F}\left[A^{2}\right]\right] .
$$

We now consider the $\mathrm{CW}$ case when only a single fundamental and second-harmonic mode of the system is excited. The $\mathrm{CW}$ solution is valid whenever the pump power is below the parametric threshold and its analysis will help in determining the stability criterion. The stationary solution of the mean-field Eq. (7) satisfies $A_{0}=\sqrt{\theta_{1}} A_{\text {in }} /\left[\left(\alpha_{1}+\rho R P_{0}\right)+\right.$ $\left.i\left(\delta_{1}+\rho J P_{0}\right)\right]$, where we have defined $R=\operatorname{Re}[\hat{I}(0)], J=$ $\operatorname{Im}[\hat{I}(0)]$, and $P_{0}=\left|A_{0}\right|^{2}$ is the $\mathrm{CW}$ intracavity power. The steady-state power is a real solution of the cubic equation,

$$
\theta_{1}\left|A_{\text {in }}\right|^{2}=P_{0}\left[\left(\alpha_{1}+\rho R P_{0}\right)^{2}+\left(\delta_{1}+\rho J P_{0}\right)^{2}\right] .
$$

From Eq. (10) it is readily seen that there are no negative solutions, so that the physical requirement that the power must be positive is always satisfied. The intracavity power can exhibit a bistable behavior and have either one or three simultaneous positive solutions, depending on the values of the phase mismatch and the detuning. In case of bistability the intracavity power will have an S-shaped dependence on the pump power (see inset of Fig. 3) and the condition for this to take place is that the equation $d\left|A_{\text {in }}\right|^{2} / d P_{0}=0$ has real solutions, which is found to occur within a certain range of input pump powers, for a detuning satisfying $\delta_{1} \geqslant \delta_{+}$ if $-J>\sqrt{3} R$ or $\delta_{1} \leqslant \delta_{-}$if $J>\sqrt{3} R$, where the critical detuning values are given by $[22,23]$

$$
\delta_{ \pm}=\frac{\alpha_{1}}{J^{2}-3 R^{2}}\left[-4 R J \pm \sqrt{3}\left(R^{2}+J^{2}\right)\right]
$$

The parameter range for which bistability can occur is plotted as a shaded region in Fig. 3, assuming a cavity with a finesse of $160\left(\alpha_{1}=\pi / 160\right)$. Note that both a finite phase mismatch and a detuning of the FF with the same sign of the mismatch are necessary conditions to obtain a bistable behavior, so the plot is symmetric with respect to a reflection through the origin. This can be understood from Eq. (10) and Fig. 2 which shows that the phase mismatch introduces a Kerr-type self-phase modulation $(J \neq 0)$, that results in a nonlinear tilt of the resonance when the intracavity power is plotted as a function of the detuning as shown in Fig. 4. The maximal intracavity power is obtained when the system is on the peak of the resonance, i.e., when the nonlinear phase shift compensates the detuning so that $\delta_{1}+\rho J P_{0}=0$. Contrary to the case of Kerr comb generation, the peak intracavity power is not only proportional to the pump power $\left|A_{\text {in }}\right|^{2}$, but it displays a saturation effect, and is further reduced by the nonlinear loss for $R>0$, so that $P_{\max }=\theta_{1}\left|A_{\text {in }}\right|^{2} /\left(\alpha_{1}+\rho R P_{\max }\right)^{2}$. This is also clearly seen in Fig. 4, which shows that the peak intracavity power for parameters corresponding to the experiment in Ref. [7] and an input pump power of $0.5 \mathrm{~W}$ is substantially smaller in the phase-matched case.

Frequency comb generation in a quadratic resonator initiates through optical parametric oscillation and the growth of subharmonic fields as the $\mathrm{CW}$ solution becomes unstable above a certain power threshold. An analysis of the linear stability of the $\mathrm{CW}$ solution (10) can be accomplished by injecting the ansatz $A=A_{0}+a_{1} \exp \left(\lambda t / t_{R}+i \Omega \tau\right)+$ $a_{-1} \exp \left(\lambda^{*} t / t_{R}-i \Omega \tau\right)$ into Eq. (7) and linearizing the resulting equation around the $\mathrm{CW}$ solution. This gives the eigenvalues (cf. Ref. [11]),

$$
\lambda_{ \pm}=-\left(\alpha_{1}+i L \hat{D}_{1, o}(\Omega)+\rho P_{0}\left[\hat{I}(\Omega)+\hat{I}^{*}(-\Omega)\right]\right) \pm \sqrt{|\hat{I}(0)|^{2} \rho^{2} P_{0}^{2}-\left(\delta_{1}-L \hat{D}_{1, e}(\Omega)-i \rho P_{0}\left[\hat{I}(\Omega)-\hat{I}^{*}(-\Omega)\right]\right)^{2}}
$$

where $\quad \hat{D}_{1, e}(\Omega)=\sum_{l \geqslant 1}\left[k_{1}^{(2 l)} /(2 l) !\right] \Omega^{2 l} \quad$ and $\quad \hat{D}_{1, o}(\Omega)=$ $\sum_{l \geqslant 1}\left[k_{1}^{(2 l+1)} /(2 l+1) !\right] \Omega^{2 l+1} \quad$ represent even and odd dispersion terms, respectively. The instability growth rate is given by the real part of the eigenvalue, and is as usual independent of the odd-order dispersion terms. The first (real) parts of the eigenvalue expression correspond to linear and nonlinear losses, while the first and second terms inside the square root correspond to the OPO gain and the phase mismatch for the MI process, respectively. MI involving the growth of sidebands with frequency $\omega_{0} \pm \Omega$ occurs via OPO down-conversion of the SH field at $2 \omega_{0}$, which acts as an intermediary stage for the cascaded process. However, a competing sum-frequency generation process between the pump mode and the subharmonic sidebands will, in the absence of walk-off, limit the growth rate and suppress the development of MI [11].

Equation (12) shows that periodic perturbations grow due to walk-off induced modulational instability, at a rate determined by the nonlinear response function. In fact, since $\hat{I}(\Omega) \rightarrow 0$ as $\Omega \rightarrow \pm \infty$, we see that MI can occur in a singly resonant SHG cavity even in the complete absence of group-velocity dispersion with the asymptotic growth rate $\lambda_{+}=-\alpha_{1}+\sqrt{|\hat{I}(0)|^{2} \rho^{2} P_{0}^{2}-\delta_{1}^{2}}$ [cf. blue solid line of 


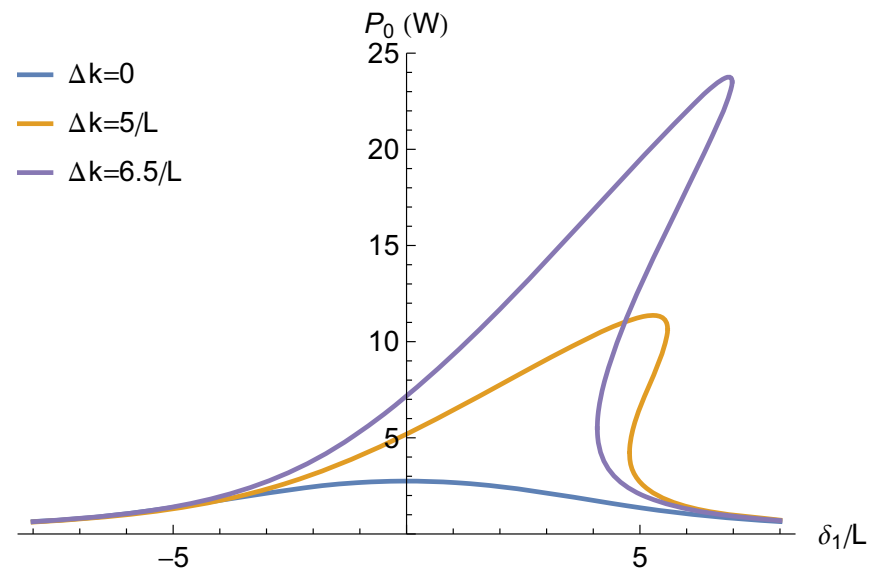

FIG. 4. Intracavity FF power as a function of normalized detuning with and without phase mismatch. The power is much larger in the former case and exhibits bistability that is seen as a nonlinear tilt of the resonance.

Fig. 5(b)]. We emphasize that this situation is fundamentally different from the case of MI in cubic nonlinear media and systems such as optical fibers, where MI occurs due to an interplay between group-velocity dispersion and the self-phase modulation effect $[24,25]$. The dispersion $\hat{D}_{1, e}(\Omega)$ at the fundamental frequency can be seen to limit the attainable gain bandwidth by providing a phase mismatch $\Delta \phi=\delta_{1}-$ $L \hat{D}_{1, e}(\Omega)-i \rho P_{0}\left[\hat{I}(\Omega)-\hat{I}^{*}(-\Omega)\right]$ at frequencies with a large offset from the pump. The MI bandwidth can be maximized by pumping close to a zero dispersion wavelength, at which point it will be limited by higher-order dispersion. Contrary to the Kerr case, the eigenvalues are generally complex and will give rise to a drift of the temporal pattern even without higher-order dispersion. The velocity of the drift depends on the imaginary part of the eigenvalue and can be influenced by odd-order dispersion terms. It may therefore be possible to compensate for it using dispersion engineering.

A simple estimate for the minimum intracavity (threshold) power required for MI to develop can be obtained by balancing the linear roundtrip loss with the OPO gain. This gives $P_{\min } \approx$ $\alpha_{1} /(\rho|\hat{I}(0)|)$, and the corresponding power of the external pump laser can be obtained from Eq. (10); cf. Ref. [23]. The frequency of the first oscillating sideband is determined by the phase-matching condition and is, in addition to the frequency dependence of the nonlinear loss introduced by the response function, generally sensitive to both the detuning as well as the temperature of the nonlinear crystal [7].

\section{RELATION TO COUPLED MODE EQUATIONS}

We will now consider the relation of the present temporal mean-field model to the frequency-domain coupled mode equations of Ref. [7]. We first show that the components of the roundtrip fundamental and second-harmonic fields $A_{m}$ and $B_{m}$ satisfy the same single-pass evolution equations as in that paper. For notational convenience we will in the following suppress the roundtrip index $m$. Inserting the modal expansions,

$$
\begin{gathered}
A_{m}=\sum_{\mu} A_{\mu}(z) \exp \left[i\left(K_{A, \mu} z-\Omega_{A, \mu} \tau\right)\right], \\
B_{m}=\sum_{\nu} \sqrt{2} B_{\nu}(z) \exp \left[i\left(K_{B, \nu} z-\Omega_{B, \nu} \tau\right)\right],
\end{gathered}
$$

into the stationary reference frame versions of Eqs. (1) and (2), i.e., written out with separate group-velocity terms, and projecting onto each frequency component we find that

$$
\begin{aligned}
\dot{A}_{\mu}= & {\left[-\frac{\alpha_{c 1}}{2}+i\left(k_{1}^{(1)} \Omega_{A, \mu}+\hat{D}_{1}\left(\Omega_{A, \mu}\right)-K_{A, \mu}\right)\right] A_{\mu} } \\
& +i \sqrt{2} \kappa \sum_{\rho, \sigma} B_{\rho} A_{\sigma}^{*} e^{i\left(K_{B, \rho}-K_{A, \sigma}-K_{A, \mu}-\Delta k\right) z} \\
\dot{B}_{v}= & {\left[-\frac{\alpha_{c 2}}{2}+i\left(k_{2}^{(1)} \Omega_{B, \nu}+\hat{D}_{2}\left(\Omega_{B, v}\right)-K_{B, \nu}\right)\right] B_{v} } \\
& +i \frac{\kappa}{\sqrt{2}} \sum_{\rho, \sigma} A_{\rho} A_{\sigma} e^{i\left(K_{A, \rho}+K_{A, \sigma}-K_{B, v}+\Delta k\right) z},
\end{aligned}
$$

where the dot is shorthand for slow-time $(t)$ derivatives and the summations should be carried out over frequencies that satisfy the relations $\Omega_{A, \mu}=\Omega_{B, \rho}-\Omega_{A, \sigma}$ and $\Omega_{B, v}=\Omega_{A, \rho}+\Omega_{A, \sigma}$, respectively. Assuming that the modes have an equidistant frequency spacing $\Omega$ we may take

$$
\begin{aligned}
& K_{A, \mu}=k_{1}^{(1)} \Omega_{A, \mu}+\hat{D}_{1}\left(\Omega_{A, \mu}\right)=k_{1, \mu}-k_{1}, \\
& K_{B, v}=k_{2}^{(1)} \Omega_{B, \nu}+\hat{D}_{2}\left(\Omega_{B, v}\right)=k_{2, v}-k_{2}, \\
& \Omega_{A, \mu}=\Omega \mu \approx \omega_{\mu}-\omega_{0}, \quad \Omega_{B, v}=\Omega v \approx \omega_{v}-2 \omega_{0},
\end{aligned}
$$

with $\mu, \nu$ being integer mode numbers and $k_{1, \mu}, k_{2, \nu}$ wave vectors corresponding to the propagation constants of the respective sideband frequencies. Defining $\hat{\kappa}=\sqrt{2} \kappa$ and using the phase-mismatch relation $\Delta k=2 k_{1}-k_{2}$ we find that the above equations reduce to

$$
\begin{aligned}
& \dot{A}_{\mu}=-\frac{\alpha_{c 1}}{2} A_{\mu}+i \hat{\kappa} \sum_{\substack{\rho, \sigma \\
\mu=\rho-\sigma}} B_{\rho} A_{\sigma}^{*} e^{i\left(k_{2, \rho}-k_{1, \sigma}-k_{1, \mu}\right) z}, \\
& \dot{B}_{\nu}=-\frac{\alpha_{c 2}}{2} B_{v}+i \frac{\hat{\kappa}}{2} \sum_{\substack{\rho, \sigma \\
\nu=\rho+\sigma}} A_{\rho} A_{\sigma} e^{-i\left(k_{2, \nu}-k_{1, \rho}-k_{1, \sigma}\right) z},
\end{aligned}
$$

which in the absence of intrinsic losses $\left(\alpha_{c i} \rightarrow 0\right)$ are equivalent to the complex conjugate of the single-pass equations presented in Table I of Ref. [7].

To derive a mean-field model for the coupled mode equations we ignore absorption losses in the following and consider them instead to be included in the coupling loss coefficient. Following a procedure similar to the continuous derivation in the previous section, the mean-field solution for the SH field is found by neglecting the $z$ variation of the FF over the duration of the roundtrip and is given by the convolution,

$$
B_{v}=i \frac{\hat{\kappa}}{2} \sum_{\rho} A_{\rho} A_{v-\rho} \int_{0}^{z} e^{-i \xi_{\rho, v-\rho} z^{\prime}} d z^{\prime},
$$

cf. Eq. (5), where $\xi_{\rho, \sigma}=k_{2, \rho+\sigma}-k_{1, \rho}-k_{1, \sigma}$. Substituting this result into the equation for the fundamental field, we find 
that

$$
\dot{A}_{\mu}=-\frac{\hat{\kappa}^{2}}{2} \sum_{\substack{\rho, \sigma \\ v=\mu+\sigma-\rho}} A_{\rho} A_{v} A_{\sigma}^{*} e^{i \xi_{\mu, \sigma} z} \int_{0}^{z} e^{-i \xi_{\rho, v} z^{\prime}} d z^{\prime},
$$

where $v=v-\rho$. Applying now the boundary condition Eq. (3) to each individual frequency component and performing the averaging over the roundtrip time yields the mean-field equation,

$$
\begin{aligned}
\frac{d a_{\mu}}{d t}= & -(\gamma+i \Delta) a_{\mu}-g_{0} \sum_{\substack{\rho, \sigma \\
v=\mu+\sigma-\rho}} \eta_{\mu \sigma \rho v} a_{\rho} a_{v} a_{\sigma}^{*} \\
& +\delta_{\mu, 0} \sqrt{2 \gamma / t_{R}} A_{\text {in }},
\end{aligned}
$$

where the cavity field at roundtrip $m$ is given by $a_{\mu}\left(t=m t_{R}\right)=$ $A_{\mu}^{(m)}(z=0)$, the slow time $t$ derivative is defined by the relation $d a_{\mu} / d t \approx\left[A_{\mu}^{(m+1)}(0)-A_{\mu}^{(m)}(0)\right] / t_{R}$, and $\delta_{\mu, 0}$ is the Kronecker delta. Additionally, we have introduced the cavity loss $\gamma=$ $\theta_{1} / 2 t_{R}$, the detuning $\Delta=\delta_{1} / t_{R}$, the nonlinear coefficient $g_{0}=$ $(\hat{\kappa} L)^{2} / 4 t_{R}$, and the coupling coefficient,

$$
\begin{aligned}
\eta_{\mu \sigma \rho v} & =\frac{2}{L^{2}} \int_{0}^{L} \int_{0}^{z} e^{i\left(\xi_{\mu, \sigma} z-\xi_{\rho, v} z^{\prime}\right)} d z^{\prime} d z \\
& =\frac{2}{L^{2}} \int_{0}^{L} e^{i \xi_{\mu, \sigma} z} \frac{1-e^{-i \xi_{\rho, v} z}}{i \xi_{\rho, v}} d z
\end{aligned}
$$

Equation (21) is, baring notation, equivalent to the complex conjugate of the general system of coupled mode equations previously derived by a heuristic method in Ref. [23]. Modedependent cavity losses and detunings can be included by generalization of Eq. (3). The special case of Ref. [7] is obtained in the three-wave approximation when only the pump frequency and two sideband modes are considered in the model. These results demonstrate that the evolution equations of the modal expansion approach can be obtained from the map given by Eqs. (1)-(4). However, it does not necessarily follow that the two mean-field approximations are exactly equivalent.

To establish the relation between the temporal mean-field Eq. (7) and the previously derived frequency-domain coupled mode equations above, we introduce the modal expansion,

$$
A=\sum_{\mu} a_{\mu}(t) \exp \left[i\left(\zeta_{\mu} t-\omega_{\mu} \tau\right)\right]
$$

into Eq. (7) and proceed analogously to the previous derivation by projecting the field onto each frequency component to obtain

$$
\begin{aligned}
t_{R} \frac{d a_{\mu}}{d t}= & {\left[-\alpha_{1}-i \delta_{1}+i\left(L \hat{D}_{1}\left(\omega_{\mu}\right)-t_{R} \zeta_{\mu}\right)\right] a_{\mu} } \\
& -(\kappa L)^{2} \sum_{\nu, \rho, \sigma} a_{\rho} a_{\nu} a_{\sigma}^{*} e^{i\left(\zeta_{\rho}+\zeta_{\nu}-\zeta_{\sigma}-\zeta_{\mu}\right) t} \hat{I}\left(\omega_{\rho}+\omega_{\nu}\right) \\
& +\delta_{\omega_{\mu}, 0} \sqrt{\theta_{1}} A_{\text {in }} e^{-i \zeta_{\mu} t}
\end{aligned}
$$

together with the energy conservation requirement given by the frequency condition $\omega_{\mu}=\omega_{\nu}+\omega_{\rho}-\omega_{\sigma}$. Assuming that
$\zeta_{\mu}=\left(L / t_{R}\right) \hat{D}_{1}\left(\omega_{\mu}\right)$ and $\omega_{\mu}=\Omega \mu$ Eq. (24) simplifies to

$$
\begin{aligned}
\frac{d a_{\mu}}{d t}= & -\left(\alpha_{1} / t_{R}+i \Delta\right) a_{\mu}-2 g_{0} \sum_{\substack{\rho, \sigma \\
v=\mu+\sigma-\rho}} \hat{I}[\Omega(\mu+\sigma)] a_{\rho} a_{\nu} a_{\sigma}^{*} \\
& \times e^{i\left(L / t_{R}\right)\left[\hat{D}_{1}(\Omega \rho)+\hat{D}_{1}(\Omega v)-\hat{D}_{1}(\Omega \sigma)-\hat{D}_{1}(\Omega \mu)\right] t} \\
& +\delta_{\mu, 0} \sqrt{2 \gamma / t_{R}} A_{\text {in }} .
\end{aligned}
$$

The two systems of coupled mode Eqs. (21) and (25) can be seen to represent the same system in the limit situation where absorption losses are neglected, i.e., $\alpha_{1} / t_{R} \rightarrow \gamma$. However, the nonlinear coupling coefficients still differ somewhat due to the fact that the contribution to the propagation constant from the group-velocity dispersion at the fundamental frequency has not been averaged in Eq. (25). Particularly, since the mode numbers should satisfy $\mu+\sigma=\rho+v$ we have

$$
\begin{aligned}
i \xi_{\mu, \sigma} & =(\hat{k}[\Omega(\mu+\sigma)]-i \Delta k)+\left(\alpha_{c 2} / 2\right)-\hat{D}_{1}(\Omega \mu)-\hat{D}_{1}(\Omega \sigma), \\
i \xi_{\rho, v} & =(\hat{k}[\Omega(\mu+\sigma)]-i \Delta k)+\left(\alpha_{c 2} / 2\right)-\hat{D}_{1}(\Omega \rho)-\hat{D}_{1}(\Omega v),
\end{aligned}
$$

which, comparing Eqs. (8) and (22), shows that $\eta_{\mu \sigma \rho v}=$ $2 \hat{I}[\Omega(\mu+\sigma)]$ and that the modal equations are identical only if second- and higher-order group-velocity dispersion at the fundamental frequency and absorption losses at the second harmonic are neglected.

Because of the difference in averaging of the FF dispersion, the two approaches will generally give slightly different results. We thus made a comparison of the eigenvalues predicted by the temporal mean-field model and the modal expansion approach. Modeling the frequency dependence of the refractive index with a Sellmeier equation for $\mathrm{LiNbO}_{3}$ and assuming quasiphase matching, we found as shown in Fig. 5(a) that the two approaches give almost identical MI growth rates for parameters corresponding to the experiment in Ref. [7]. To highlight the difference, a comparison of the MI gain over a wide bandwidth in the absence of a phase mismatch $\Delta \phi$ for the MI process [i.e., assuming that the expression in parentheses under the square root of Eq. (12) vanishes] is also shown in Fig. 5(b). The distinction between the temporal mean-field model (blue) and the modal expansion approach (yellow, dashed), is that the OPO gain in the latter case depends on the group-velocity dispersion and has a finite bandwidth. However, this effect is negligible for realistic systems where the effects of chromatic dispersion are overshadowed by the action of temporal walk-off. The two approaches are in excellent agreement when considering the full MI gain and the difference between them are therefore minor in practice. Note also that fast Fourier transform techniques cannot be used to speed up numerical simulations of the coupled mode equations, unless the nonlinear coupling between the fields in Eq. (21) can be written as a convolution [26]. However, Eq. (21) cannot in general be written as a single convolution, which increases the computational complexity in comparison to Eq. (7).

\section{COMB DYNAMICS}

To begin characterizing the comb dynamics, we show in Fig. 6 two plots of the spectral evolution of the comb 

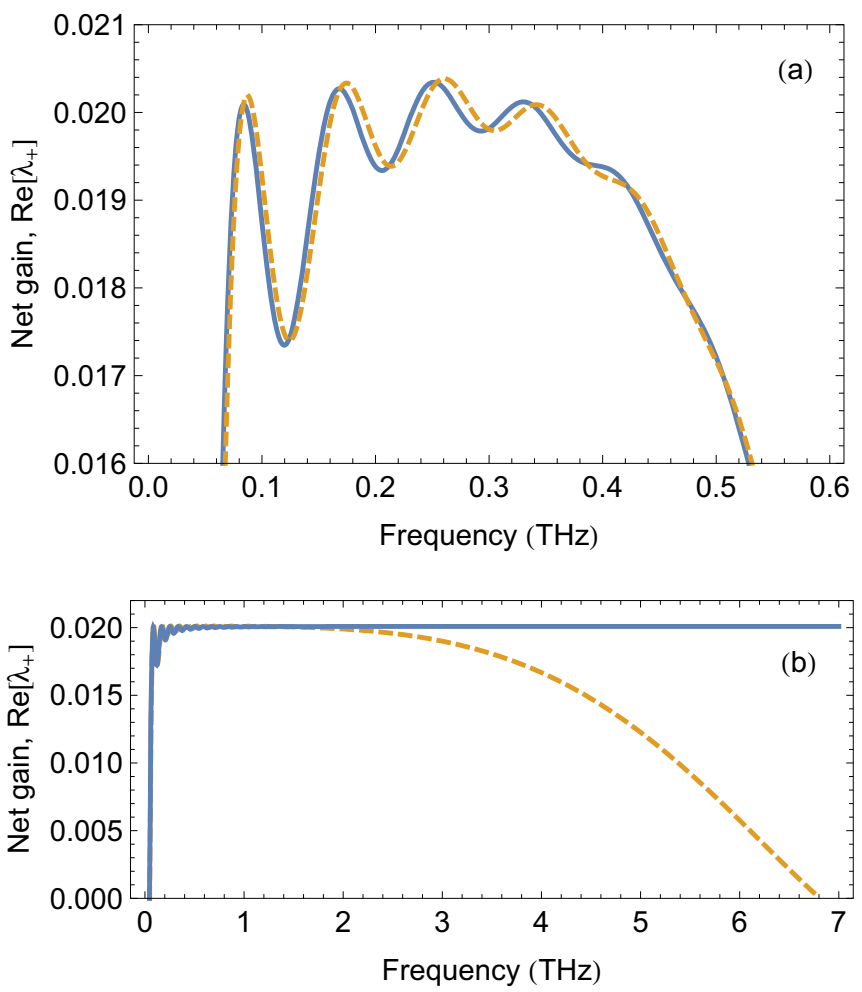

FIG. 5. Comparison of MI gain predicted by the temporal meanfield model (blue, solid line) and the modal expansion approach (yellow, dashed). (a) Full MI gain. (b) MI gain when neglecting the phase mismatch $\Delta \phi$ for the MI process. The gain of the temporal mean-field model does not account for frequency dependence of the OPO gain and is seen to be asymptotic to a constant value in this case.

states obtained during a slow sweep of the detuning, which experimentally corresponds to a sweep of the laser frequency across the resonance. This procedure is similar to a technique commonly used for the excitation of cavity soliton states in Kerr resonators [27,28]. An analogous study for a doubly resonant cavity has also been presented in Ref. [14], where multiple dynamical regimes and abrupt shifts in the repetition rate of the temporal MI pattern were numerically observed. The parameters for the simulations are given in the caption and correspond to those of the experiment in Ref. [7]. Although $\mathrm{MI}$ is induced by the temporal walk-off, the group-velocity dispersion is still important for determining the comb bandwidth. To highlight the dependence on dispersion, we consider identical parameters but different signs for the second-order FF dispersion coefficient in Figs. 6(a) and 6(b), while neglecting dispersion terms of higher order. We linearly vary the detuning between $\delta_{1} \in[-0.1,0.2]$ and $\delta_{1} \in[-0.2,0.1]$, respectively, over a time scale of 2 million roundtrips $(\sim 4$ $\mathrm{ms}$ ) and record the instantaneous spectras that correspond to vertical cross sections of the figure. Also plotted in the figures are white dashed contour lines bounding the regions where Eq. (12) predicts that MI can occur, and solid white lines giving the frequencies for the peak MI gain. The variation of the intracavity power for the $\mathrm{CW}$ state is further shown below the figures. By comparing Figs. 6(a) and 6(b), we can see that similar dynamics can be observed both in the normal and the anomalous dispersion regimes, and that the
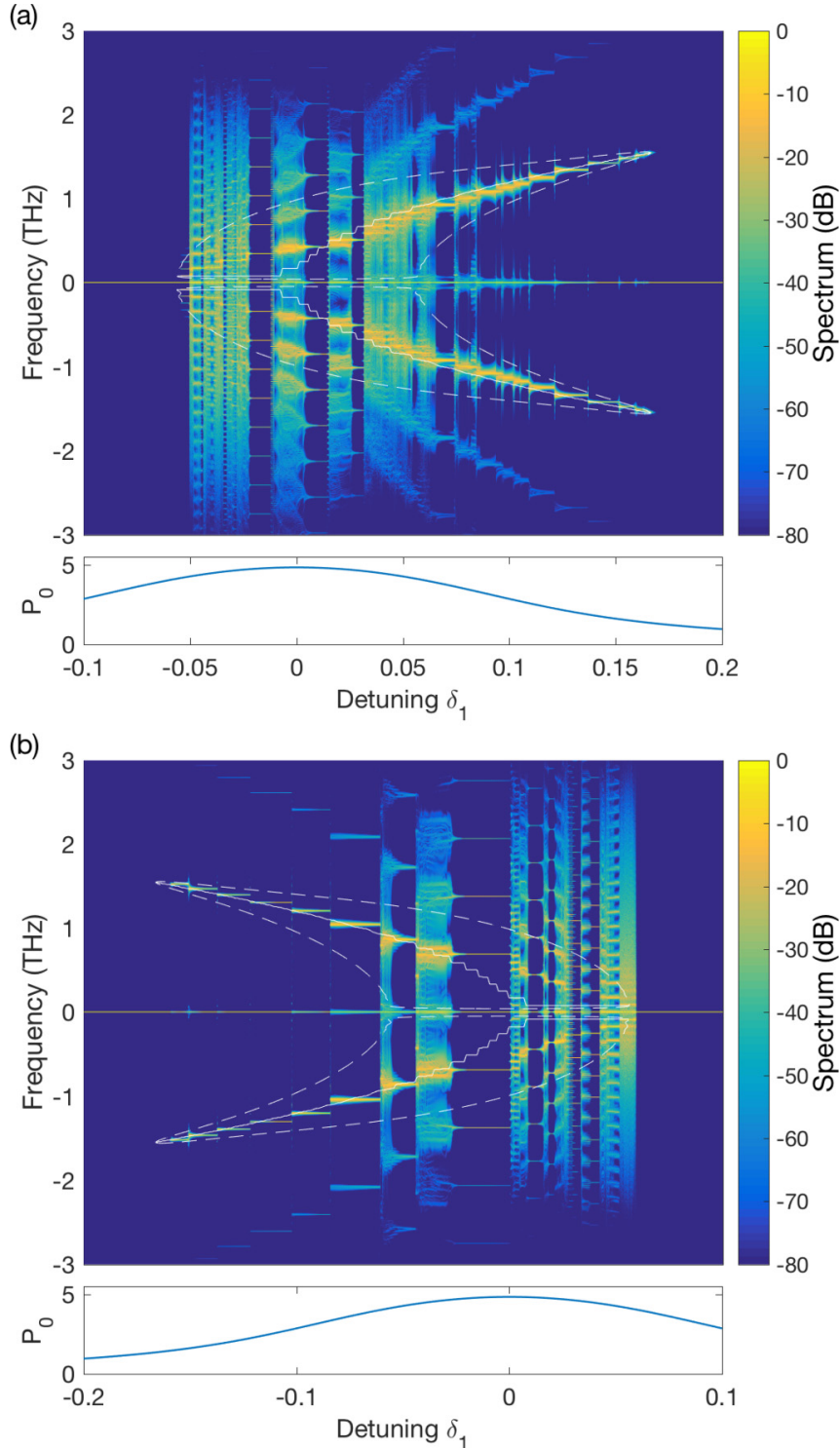

FIG. 6. Influence of the detuning on the spectral dynamics. Density plot of the normalized spectrum and variation of the CW intracavity power. (a) Normal dispersion $k_{1}^{(2)}>0$. (b) Anomalous dispersion $k_{1}^{(2)}<0$. Parameters corresponding to the experiment in Ref. [7]: $P_{\text {in }}=2 \mathrm{~W}, \theta_{1}=\alpha_{1}=\pi / 160, L=15 \mathrm{~mm}, \kappa=11.41 \mathrm{~W}^{-1 / 2} \mathrm{~m}^{-1}$, $\Delta k=0, \quad \Delta k^{\prime}=792 \mathrm{ps} / \mathrm{m}, \quad\left|k_{1}^{(2)}\right|=0.234 \mathrm{ps}^{2} / \mathrm{m}, \quad$ and $k_{2}^{(2)}=$ $0.714 \mathrm{ps}^{2} / \mathrm{m}$.

two are roughly mirror images of each other with respect to the detuning. This behavior can also be seen directly from the eigenvalue Eq. (12) which predicts the MI gain. When the walk-off dominates the dispersion at the second harmonic, i.e., when $\left|\Delta k^{\prime}\right| \gg\left|k_{2}^{(2)} \Omega\right| / 2$, the nonlinear response function will approximately satisfy $\hat{I}\left(\Omega_{0}+\Omega\right) \approx \hat{I}^{*}\left(\Omega_{0}-\Omega\right)$ with $\Omega_{0}=\Delta k / \Delta k^{\prime}$. Consequently for $\Delta k=0$ we have that the bandwidth limiting phase mismatch for the MI process $\Delta \phi$ becomes independent of the nonlinear contribution and reduces to $\left[\delta_{1}-\left(k_{1}^{(2)} L / 2\right) \Omega^{2}\right]^{2}=\left[\left(-\delta_{1}\right)-\left(-k_{1}^{(2)} L / 2\right) \Omega^{2}\right]^{2}$. Spectral dynamics similar to a normal dispersion resonator with positive detuning can be thus be obtained by operating a resonator having anomalous dispersion with negative 
detuning. From the simulations of Fig. 6 we can see that the parameter range for the instability is well predicted by the MI analysis, and that a variety of both stable and unstable comb states with different comb line spacings are possible. Particularly, in the normal dispersion region with $\delta_{1}>0.07$ and the anomalous dispersion region with $\delta_{1}<$ -0.07 we see discrete jumps in the sideband spacing as the detuning is varied. This behavior can be understood to occur because the maximum MI gain frequency (solid white line) switches between different minima of the nonlinear loss; cf. Fig. 2.

To better understand the comb formation dynamics for fixed parameters, we consider in Fig. 7 an example of the spectral evolution of the fundamental field for a characteristic set of parameters. We perform a numerical simulation of Eq. (7) with the detuning held fixed and record the instantaneous spectrum over a period of 10000 roundtrips $(\sim 20 \mu \mathrm{s})$. The top plot of the figure shows the temporal pattern obtained at the end of the simulation, and below it is shown the stationary spectrum together with the initial MI growth rate predicted by Eq. (12). Following the evolution from the beginning, we see that the CW pump mode becomes modulationally unstable and quickly generates a broad spectrum involving a large
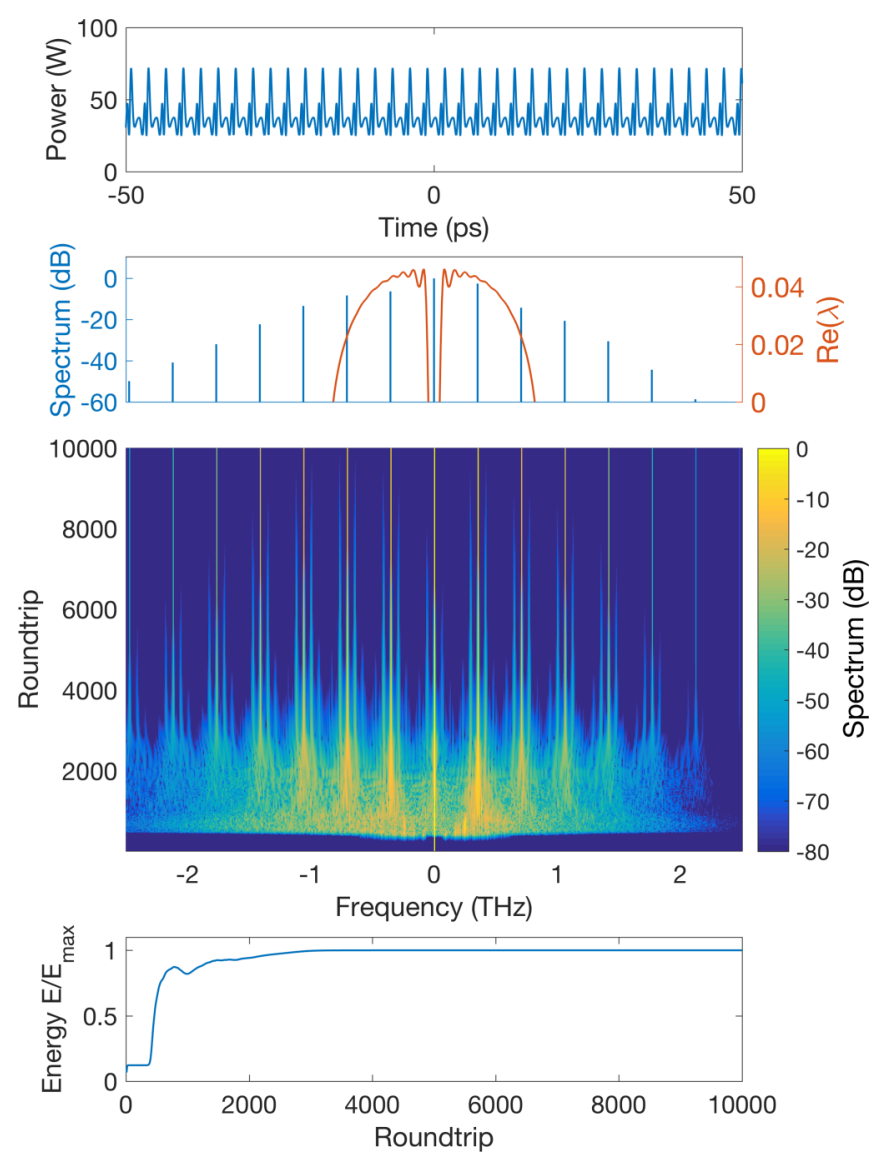

FIG. 7. Spectral evolution of the fundamental field (large panel). Top plot shows temporal profile, below it is shown the final spectrum and the initial MI growth rate, while the bottom plot shows evolution of the dimensionless intracavity energy. The parameters are the same as in Fig. 6(a) with $\delta_{1}=-0.02$. number of cavity modes with the fundamental spacing of a single free-spectral range (FSR). Since these modes originate from amplified noise, they are however not phase locked, which manifests itself by a turbulent evolution with a random scrambling of phases and large amplitude fluctuations of the individual modes. After roughly 2000 roundtrips a few modes spaced by 39 FSRs, which is close to the middle of the MI gain spectrum near its fourth highest peak, become phase locked, and start to dominate the spectra. As time progresses we find that additional harmonics further away from the pump mode also lock. At this point the remaining modes are no longer experiencing net parametric gain and start to decay from absorption losses. We see that most modes decay rather quickly, but that a pair of subcomb sidebands around each mode of the main comb sees a smaller net loss and persists for a few thousand roundtrips before eventually disappearing and leaving behind a stationary, slightly asymmetric, frequency comb that corresponds to a slowly drifting temporal pattern (top panel of Fig. 7). Interestingly, the stationary solution is seen to represent a maximum for the intracavity energy (bottom panel of Fig. 7). The comb is consequently more energetic in the final multiple FSR state, with larger amplitude of the individual comb lines, than in the MI unstable regime where the spectrum is much denser. The overall dynamics of the formation process is similar to that of Kerr cavity solitons and resembles the self-organizing phase synchronization mechanism of the Kuramoto model [29].

\section{CONCLUSIONS}

In conclusion, we have considered frequency comb generation in a singly resonant second-harmonic-generation cavity system containing a quadratically nonlinear and temporally dispersive medium. Starting from an infinite-dimensional map of nonlinear evolution equations for the fundamental and second-harmonic fields, we have derived an accurate dynamical model featuring a single mean-field equation with a noninstantaneous nonlinear response function. The origin of the nonlinear response is found to be phase-matched cascaded $\chi^{(2)}$ processes that lead to an effective cubic nonlinearity and allows modulational instability to occur through an interplay of nonlinearity and the difference in group velocity between the pump frequency and its second harmonic. We have further shown that the mean-field model can be related to a frequency-domain modal expansion approach where the dynamics of the system is governed by coupled mode equations for the slow time evolution of each individual mode. The two approaches are found to involve different mean-field averaging procedures, but to be approximately equivalent in general, with equality in the limit that absorption losses and group-velocity dispersion at the fundamental frequency can be neglected. Results of numerical simulations have also been presented, illustrating the range of different comb states that can be generated in both normally and anomalously dispersive quadratic resonator systems, together with an example showing typical comb formation dynamics for a mode-locked temporal pattern. 


\section{ACKNOWLEDGMENTS}

This work was funded by the Swedish Research Council (Grant No. 2013-7508), the Italian Ministry of University and Research (MIUR) (PRIN 2015KEZNYM (NEMO) and Progetto Premiale QUANTOM - Quantum Opto-Mechanics), the Rutherford Discovery Fellowships of the Royal Society of New Zealand, and the Marsden Fund of the Royal Society of New Zealand.

\section{APPENDIX: NORMALIZATION}

To eliminate superfluous parameters and make the variables take on values of order unity it is convenient to express the mean-field Eq. (7) in dimensionless form. This can, e.g., be done by introducing the choice of normalization $u=A \kappa L / \sqrt{\alpha_{1}}, T=\tau \sqrt{2 \alpha_{1} /\left(\left|k_{1}^{\prime \prime}\right| L\right)}$, and $\xi=t \alpha_{1} / t_{R}$. If dispersion beyond the second order is neglected, the mean-field equation then takes on the form,

$$
\begin{aligned}
\frac{\partial u(\xi, T)}{\partial \xi}= & {\left[-1-i \Delta_{1}-i \eta_{1} \frac{\partial^{2}}{\partial T^{2}}\right] u } \\
& -u^{*}\left[u^{2}(\xi, T) \otimes I(T)\right]+S,
\end{aligned}
$$

where we have defined $\Delta_{1}=\delta_{1} / \alpha_{1}, \quad \eta_{1}=\operatorname{sign}\left(k_{1}^{\prime \prime}\right)$, and $S=\kappa L \sqrt{\theta_{1} / \alpha_{1}^{3}} A_{\text {in }}$. The nonlinear response function $I(T)=$ $\mathscr{F}^{-1}[\hat{I}(\Omega)]$ is the same as in Eq. (8), i.e.,

$$
\hat{I}(\Omega)=\frac{1-e^{-i x}-i x}{x^{2}}
$$

but depends on $x(\Omega)=\Delta k L+i \hat{k}(\Omega) \alpha_{1}$ and $\hat{k}(\Omega)=-\mu_{2}+$ $i\left[\eta_{2} \Omega^{2}+d \Omega\right]$, with the dimensionless parameters $\mu_{2}=$ $\alpha_{c 2} L /\left(2 \alpha_{1}\right), \eta_{2}=k_{2}^{\prime \prime} /\left|k_{1}^{\prime \prime}\right|$, and $d=\Delta k^{\prime} \sqrt{2 L /\left(\alpha_{1}\left|k_{1}^{\prime \prime}\right|\right)}$.
[1] P. Del'Haye, A. Schliesser, O. Arcizet, T. Wilken, R. Holzwarth, and T. J. Kippenberg, Nature (London) 450, 1214 (2007).

[2] T. J. Kippenberg, R. Holzwarth, and S. A. Diddams, Science 332, 555 (2011).

[3] T. J. Kippenberg, S. M. Spillane, and K. J. Vahala, Phys. Rev. Lett. 93, 083904 (2004).

[4] A. A. Savchenkov, A. B. Matsko, D. Strekalov, M. Mohageg, V. S. Ilchenko, and L. Maleki, Phys. Rev. Lett. 93, 243905 (2004).

[5] V. Ulvila, C. R. Phillips, L. Halonen, and M. Vainio, Opt. Lett. 38, 4281 (2013).

[6] V. Ulvila, C. R. Phillips, L. Halonen, and M. Vainio, Opt. Express 22, 10535 (2014).

[7] I. Ricciardi, S. Mosca, M. Parisi, P. Maddaloni, L. Santamaria, P. De Natale, and M. De Rosa, Phys. Rev. A 91, 063839 (2015).

[8] S. Schiller, G. Breitenbach, R. Paschotta, and J. Mlynek, Appl. Phys. Lett. 68, 3374 (1996).

[9] S. Schiller, R. Bruckmeier, and A. G. White, Opt. Commun. 138, 158 (1997).

[10] G. I. Stegeman, D. J. Hagan, and L. Torner, Opt. Quant. Electron. 28, 1691 (1996).

[11] F. Leo, T. Hansson, I. Ricciardi, M. De Rosa, S. Coen, S. Wabnitz, and M. Erkintalo, Phys. Rev. Lett. 116, 033901 (2016).

[12] P. Lodahl and M. Saffman, Phys. Rev. A 60, 3251 (1999).

[13] M. Bache, O. Bang, J. Moses, and F. W. Wise, Opt. Lett. 32, 2490 (2007).

[14] F. Leo, T. Hansson, I. Ricciardi, M. De Rosa, S. Coen, S. Wabnitz, and M. Erkintalo, Phys. Rev. A 93, 043831 (2016).

[15] L. A. Lugiato and R. Lefever, Phys. Rev. Lett. 58, 2209 (1987).
[16] S. Coen, H. G. Randle, T. Sylvestre, and M. Erkintalo, Opt. Lett. 38, 37 (2013).

[17] A. V. Buryak, P. D. Trapani, D. V. Skryabin, and S. Trillo, Phys. Rep. 370, 63 (2002).

[18] T. Hansson, F. Leo, M. Erkintalo, J. Anthony, S. Coen, I. Ricciardi, M. De Rosa, and S. Wabnitz, J. Opt. Soc. Am. B 33, 1207 (2016).

[19] M. Haelterman, S. Trillo, and S. Wabnitz, Opt. Commun. 91, 401 (1992).

[20] R. H. Stolen, W. J. Tomlinson, H. A. Haus, and J. P. Gordon, J. Opt. Soc. Am. B 6, 1159 (1989).

[21] C. Conti, M. A. Schmidt, P. S. J. Russell, and F. Biancalana, Phys. Rev. Lett. 105, 263902 (2010).

[22] A. G. White, J. Mlynek, and S. Schiller, Europhys. Lett. 35, 425 (1996).

[23] S. Mosca, I. Ricciardi, M. Parisi, P. Maddaloni, L. Santamaria, P. De Natale, and M. De Rosa, Nanophotonics 5, 316 (2016).

[24] G. Agrawal, Nonlinear Fiber Optics, 5th ed. (Academic Press, New York, 2012).

[25] T. Hansson, D. Modotto, and S. Wabnitz, Phys. Rev. A 88, 023819 (2013).

[26] T. Hansson, D. Modotto, and S. Wabnitz, Opt. Commun. 312, 134 (2014).

[27] T. Herr, V. Brasch, J. D. Jost, C. Y. Wang, N. M. Kondratiev, M. L. Gorodetsky, and T. J. Kippenberg, Nat. Photon. 8, 145 (2013).

[28] K. Luo, J. K. Jang, S. Coen, S. G. Murdoch, and M. Erkintalo, Opt. Lett. 40, 3735 (2015).

[29] Y. H. Wen, M. R. E. Lamont, S. H. Strogatz, and A. L. Gaeta, Phys. Rev. A 94, 063843 (2016). 